\title{
Frameworks for Practice
}

\author{
Robyn Stead \\ RTLB, Howick/Pakuranga Cluster
}

\section{ABSTRACT}

This study investigates the types of assessment models used by registered psychologists employed by Ministry of Education: Special Education (MOE:SE) who work in the area of severe and challenging behaviour. The aim of the study was to identify and explore frameworks for practice which are currently used at MOE:SE; the theories which underpin these as well as which aspects of the frameworks were supportive of multi-agency/multidisciplinary work.

A semi-structured interview format was used to encourage participants to discuss the frameworks and theories which they use during practice in the field. The participants were six randomly-chosen registered psychologists who work at MOE:SE.

While responses varied, the most commonly reported frameworks for practice were Effective Interventions for Behaviour Challenges, Functional Behavioural Assessment and Situational Analysis. The theories which the participants reported as underpinning their practice were also diverse, although behavioural theory was reportedly used by all participants. Collaboration was reported most often as being a supportive aspect of frameworks which were used during inter-disciplinaryl inter-agency work.

\section{INTRODUCTION}

\section{Practice Paper}

Keywords: assessment, practice frameworks, psychologist, severe behaviour, situational analysis

\section{Purpose of the study}

Intern psychologists who are preparing to join the profession are statistically most likely to find employment within MOE:SE. It is of interest to preservice psychologists to learn more about how practicing registered psychologists work within the organisation.

\section{BACKGROUND}

Ministry of Education: Group Special Education MOE:SE is the largest employer of psychologists in New Zealand according to the Psychology Workforce Annual Survey (2006). The work of Psychologists who are employed at GSE is guided by the Internal Specialist Services Standards (MOE, 2006). In her introduction to the Specialist Services Standards, Barbara Disley, Group Manager, MOE:SE, notes that the work of the professionals (this includes psychologists) is to support 'children and young people to be: present, participating, and engaged in experiences that maximise learning [and that] this work contributes to the important outcomes we are seeking: achievement, wellbeing, community and workforce participation' (p.13).

\section{Ministry of Education definition of severe and challenging behaviour \\ The Ministry of Education defines severe and challenging behaviour of children and young people as 'behaviour that may endanger themselves or others, damage property, or affect the child or young person's social interactions and learning' (Ministry of Education, Severe Behaviour Service, 2008 p.17).}

\section{In-Service training at MOE:SE}

Since 2007 GSE has engaged in an in-house nationwide training programme. The purpose of the training is to ensure that practitioners are aware of effective interventions for children and young people with severe and challenging behaviour as supported by recent research. This training programme is called Effective Interventions for Behaviour Challenges (EIBC).

The EIBC training programme has been informed by two large meta-analyses of research into severe behaviour: Meyer and Evans (2006), which focuses on severe behaviour in children and youth with developmental disabilities, and The Church Report (2003), which focuses on children who are considered to have potential for normal development and display characteristics of anti-social behaviour at an early age.

\section{Multi-agency/interdisciplinary work}

Registered psychologists working at MOE:SE are required to work with a wide range of other agencies and professionals from other disciplines. These may be other professionals who are employed by MOE:SE such as speech and language therapists, occupational therapists, early intervention teachers, special education advisors and specialists on the education of children with hearing or vision impairment. Outside agencies which work with psychologists from MOE:SE may include, but are not limited to, the police, staff from Child, Youth and 
Family Services, Resource Teachers of Learning and Behaviour, teachers, school administrators and medical professionals.

\section{Frameworks for practice}

Two influential sources of frameworks of practice in New Zealand are Group Special Education and Massey University.

\section{Ministry of Education}

The Practice Leader: Behaviour, Professional Practice Unit, Ministry of Education, has noted that 'Models of practice, frameworks etc will vary according to how the practitioner has been trained, their own experience and background and their own personal values and principles. The only "framework" required of MOE:SE behaviour practitioners is the Specialist Service Standards (SSS)' (Cull, personal communication, August 21, 2008). SSS are a guide to service delivery and are tied to the review process, Review of Individual Behaviour Service (RIBS). Together, RIBS and SSS are tools for the Ministry of Education to promote and monitor standards of service delivery. It is left to the individual practitioner to choose a model which fits with his or her particular background, values and principles.

\section{Massey University}

Massey University is currently the only provider in New Zealand of preparatory training for registration as a psychologist in the field of education. The framework, Situational Analysis, taught to pre-service psychologists, is described by Annan (2005) as a framework for practice which has both style and structure and is able to be applied with both individual and systems fieldwork. When considering Situational Analysis, style refers to the "particular theoretical position" of the practitioner, while structure refers to the 'steps taken by practitioners to gather, analyse and use information' (Annan 2005, p. 133).

\section{LITERATURE REVIEW}

The purpose of this exploratory research was to review the frameworks which were likely to be featured in discussion with the study participants.

\section{Research which informs EIBC}

Neither Meyer and Evans (2006) nor Church (2003) explicitly discuss a process for fieldwork where analysis of situations takes place before interventions are considered. Their work concentrates on intervention rather than on a broad analysis of the whole situation.

Church focuses on early identification of children with anti-social development through a screening process and the research which describes effective intervention for children identified as developing anti-socially throughout their developmental stages.
Church (2003) is quite prescriptive in his discussion of analysis stating that 'A review of research into the development of anti-social behaviour in children indicates that we now have a reasonably good understanding of the way in which anti-social development occurs' (p.3) He further notes that research has also identified:

The learning processes which need to be targeted by any intervention are the positive and negative reinforcement processes which teach, strengthen and maintain defiant, coercive and aggressive responses during interactions with other people. The contexts which need to be targeted are the child's anti-social interactions with parents and siblings (the home context), with teachers and classmates (the school context) and with peers and associates (the playground and recreational contexts) (p.3).

The implication that Church makes is that there is a highground in fieldwork from which clarity about situations can be easily gained.

Meyer and Evans (2006) have completed a meta-analysis of research into effective interventions for children and youth with developmental disabilities. They discuss the complexity of situations in which psychologists who work with children and young people demonstrating severe and challenging behaviour are involved, and note that 'there will not be a specific intervention or strategy that is demonstrated to be effective in the abstract, so that it can then be used with assurance by any reasonably skilled teacher or clinician' (p.31). Whilst they acknowledge the complexity of and review the literature on what constitutes effective intervention, they do not provide a process for practice which can be used by psychologists in the field to help them to work through the complex issues integral to the situations they engage in.

\section{Functional behavioural analysis}

Both Church (2003) and Meyer and Evans (2006) state that Functional Behavioural Analysis (FBA) is one of the most commonly-used procedures to determine the purposes (or intent) of behaviour. FBA is a strategy which employs many methods to gather information that indicates the reason for or function of behaviour (Gresham, Watson \& Skinner, 2001). It developed from the field of applied behaviour analysis and is the practical application of FBA which is used in experimental conditions to measure the effect of changing variables on a specific behaviour. FBA takes an environmental approach and its theoretical stance avoids locating the problem within the child. FBA provides a process or structure to enable psychologists to work through fieldwork from the initial referral through to closure of the case. 


\section{Situational analysis}

Monsen, Graham, Frederickson and Cameron's (1998)

article on problem analysis relates to Annan's (2005)

article on situational analysis as they both present

frameworks for practice which psychologists engaged

in fieldwork can use. It is helpful to look at Monsen et

al.'s (1998) work in order to gain perspective on Annan's (2005) situational analysis.

Monsen et al. (1998) report that fieldwork is often complex, confusing and deals with situations which have been in existence for some time and have already proved resistant to intervention. Monsen et al.'s article about problem analysis discusses the difficulty experienced by psychologists who work in the field in finding situations which can be clearly defined, with interventions easily flowing from the definition of the problem.

Monsen et al. (1998) propose that a problem analysis framework allows practitioners to explicitly demonstrate their reasoning, application of theory and rationale for specific data gathering, as well as the conceptual links between these areas of practice. This means that the practitioner's work is transparent, and can be challenged or supported. Importantly it allows the practitioner to avoid the role of "expert advice dispenser" (Monsen et al., 1998, p.239), which can leave participants feeling disengaged with the problem and unlikely to feel empowered to be part of making necessary changes.

Annan (2005) explains situational analysis as a framework which allows psychologists to 'tailor their fieldwork to the diverse situations in which they work' (p.131). Both Monsen et al. (1998) and Annan (2005) cite Robinson's (1987) problem-analysis method as being influential in the development of their frameworks. Annan (2005) particularly credits Robinson as being influential in the development of the structure of the framework.

Situational analysis has both style and structure. It is the style of the framework which differentiates it from the work of both Robinson (1987) and Monsen et al. (1998) and this relates to the 'particular theoretical position taken in the ascription of meaning and the construction of new solutions' (Annan, 2005, p.133). The particular theoretical positions of situational analysis are: 'ecological, collaborative, evidence-based and constructive' (Annan, 2005, p.145).

Annan (2005) addresses the issue of evidence-based interventions which is so prominent in the meta-analysis reports of Church (2003) and Meyer and Evans (2006). She proposes that situational analysis combines both evidence from the research and the personally gained knowledge of the participants. This approach means that all participants in a situation are valued and included in a collaborative process to develop interventions which are specific to the situation.
The research reviewed has highlighted that EIBC and FBA are focused on intervention while situational analysis provides both a style and structure for addressing casework from referral to closure.

\section{METHOD}

Negotiation: An initial request was made to a district manager of a regional area at MOE:SE for permission to interview registered psychologists about their work with children and young people with severe and challenging behaviour. A project proposal was developed after this initial discussion.

The project proposal was taken to a meeting of district managers and discussed. The lead practitioner for behaviour in the area agreed to coordinate the interviews and contacted the intern psychologist to discuss the details of data collection.

The lead practitioner for behaviour organised a day of meetings at both office locations for the intern psychologist to conduct the interviews with the volunteer participants. The intern psychologist also had the opportunity to meet some of the other staff who work with registered psychologists. It was agreed that all interview notes would be typed and sent to the participants for checking and modifying and that the final report would be sent to the lead practitioner subsequent to handing in to the university for marking.

Participants: Participants included six registered psychologists employed at MOE:SE, in a regional area, spread across two offices. These psychologists volunteered after being invited to participate in the study via an email sent by the lead practitioner for behaviour. Participants were invited to take part in a study about the frameworks they used in fieldwork with children with severe and challenging behaviours which would be undertaken by an intern psychology student from Massey University. All participants signed informed consent forms and an organisational consent form was also signed by the lead practitioner for behaviour.

There were five females and one male. Ages ranged from 25-35 years to $50+$ years with a mode of $50+$ years. Length of service in MOE:SE as a registered psychologist ranged from 10 months to 15 years with a mean length of service of 6.5 years, case loads ranged from 15 to 36 students seen within one term with a mean of 25 cases seen per term by a psychologist. 
Procedure: An interview schedule was developed which contained 10 questions relating to frameworks for practice as well as information about age ranges, length of service, gender of practitioners, numbers of children or young people typically seen and kinds of behaviours encountered in fieldwork. Questions were a mix of open and closed and were chosen to elicit information about how practitioners worked in the field, allowing them to choose their own words to describe their personal experience.

Each participant was interviewed individually with a scheduled interview time of one hour. Questions were asked in order with the intern psychologist making notes as replies were given. Clarifying questions were sometimes asked by the intern psychologist or participants as they felt necessary. The interview tone was conversational. Copies of the interviewer's notes were emailed to participants to check for accuracy. Data was collated and organised according to emerging common themes.

\section{RESULTS}

Kinds of behavioural concerns referred to registered psychologists at MOE:SE:

There were 14 different reasons for referral reported by the six registered psychologists with violent verbal or physical behaviour being reported by five of the six participants as a reason for referral; the next two most commonly mentioned were anxiety and non-compliance, with all other reasons being reported one time (see Chart A).

\begin{tabular}{|ll|}
\hline Anxiety & $2 / 6$ \\
\hline Violent verbal or physical behaviour & $5 / 6$ \\
\hline Task avoidance & $1 / 6$ \\
\hline Non compliance & $2 / 6$ \\
\hline Mis-match of participants perceptions & $1 / 6$ \\
\hline Property damage & $1 / 6$ \\
\hline Difficulty with communication & $1 / 6$ \\
\hline Self regulation & $1 / 6$ \\
\hline Learning & $1 / 6$ \\
\hline Autism spectrum disabilities & $1 / 6$ \\
\hline Rigid thoughts & $1 / 6$ \\
\hline Deviant behaviour & $1 / 6$ \\
\hline Parenting difficulties & $1 / 6$ \\
\hline Safety of self and others & $1 / 6$ \\
\hline
\end{tabular}

Chart A: Reasons for referral

Frameworks for practice: Graph A displays the 11 different frameworks for practice reportedly used by the six registered psychologists who participated in the study. All psychologists reported that they used more than one framework for practice.
Four of the six participants used Effective Interventions for Behaviour Challenges (EIBC), Functional Behaviour Assessment (FBA) and Situational Analysis, two reported using Service Pathways, while RIBS, Te Whaariki, Developmental, Systemic, Cognitive, Behavioural and Gestalt frameworks were each mentioned once.

The psychologists who reported using the largest number of different frameworks were in the 50+ age range (Graph B). Situational Analysis was reportedly used by all of the participants in the 25-35 and 36-45 age groups and one participant in the $50+$ age group. Service Pathways were mentioned both by a psychologist in the $50+$ age range and a psychologist in the 25-35 age range. Some of the participants who reported using Situational Analysis noted that they used it particularly to organise data into dimensions leading to analysis and used FBA as a method of data collection which contributed to dimensions.
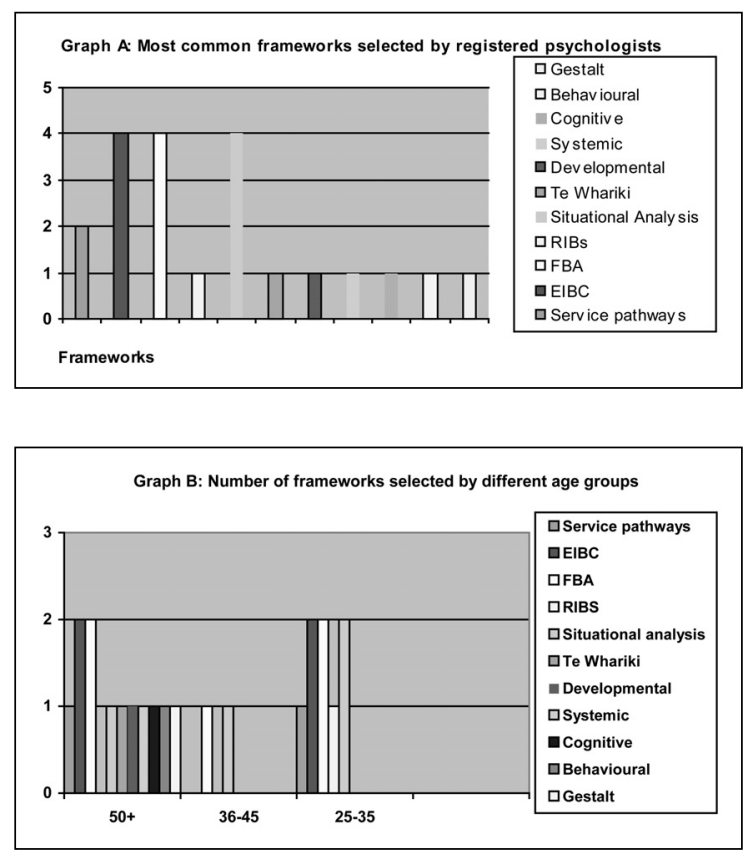

Theories which underlie practice: Graph $\mathrm{C}$ contains data about the 14 theories reported by the registered psychologists as underlying the frameworks they use. All psychologists reported behavioural theory underlying their practice. Developmental, ecological and cognitive theories were reported by five of the six psychologists. Narrative, psychometric and learning theories were reported by two psychologists each with Drawing, Attachment, Neuro-linguistic, Cognitive Behaviour Therapy, Social Learning, Solutions-Focused and SocioCultural theories each being mentioned once. 


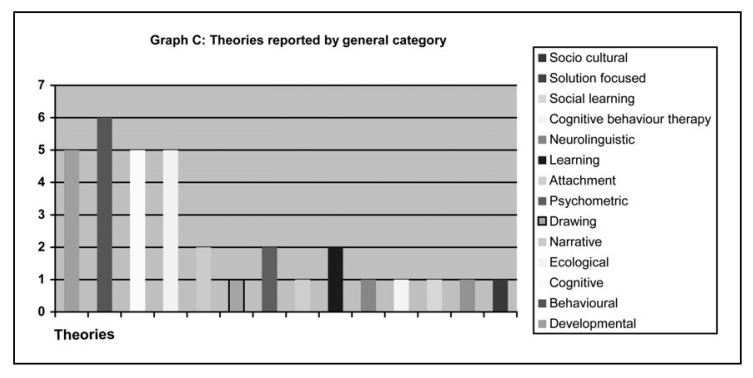

All psychologists selected a wide variety of theorists with two noting that their choice of underpinning theorists was "eclectic". The largest number of theorists selected by an individual was 11 and the smallest, three, with the mean number being six.

One of the psychologists who reported developmental theories underlying her framework for practice noted that 'it all comes back to ages and stages', and another noted that developmental theorists were helpful to underpin practice with 'children as they move age-wise [through the education system]'.

\section{Aspects of framework for practice which support} successful outcomes: Graph D shows the 12 aspects of frameworks which the participants reported as supportive of successful outcomes when working with children and young people who displayed severe and challenging behaviour. There was very little repetition of aspects by the participants. The aspects which were reported by two participants were collaboration, positive reinforcement and defining dimensions. One participant noted that collaboration got 'buy-in [which] was very important so that people don't feel dictated to'. All other aspects were reported once.

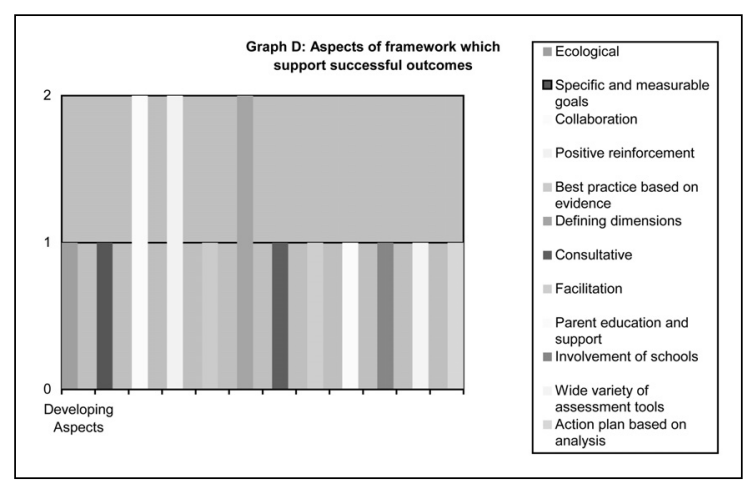

Aspects of the framework for practice that are still developing: The participants were asked "Are there any aspects of the framework of practice that are still developing?" The word any was chosen carefully so that there was no implication that the participants should, through the framing of the question, define areas which needed development.
All participants did report areas which they needed to develop as a practitioner. These are displayed in Graph E. Timeframes were mentioned twice. The participants said this was due to the new training in EIBC and a reflection on the time it takes to learn and integrate new procedures (such as filing) until they become more automatic. All other aspects: qualitative data needed to be included, cognitive aspects need to be included, section to include key barriers, effective data collection which teachers will use, paperwork, expectations to always get it right, expectations of number of children seen, new assessment tools, DSM knowledge, intra-personal characteristics such as emotions, were mentioned once. One of the more experienced registered psychologists reported that she was still learning.

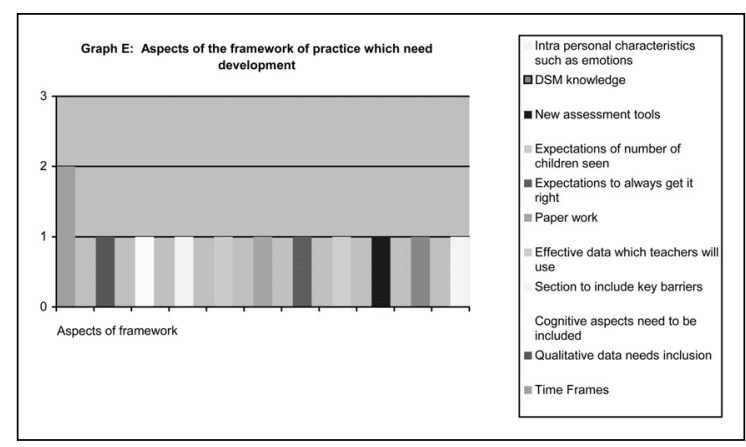

Aspects of the framework for practice which are supportive of inter-disciplinary/inter-agency work: While the processes to develop inter-disciplinary/ inter-agency interventions are often prescribed by the local and national management of MOE:SE, four of the six participants reported using regular meetings to develop their inter-disciplinary/inter-agency work. One psychologist reported that maintaining regular communication through email or phone contact was a helpful process. Another said that clearly defining and documenting roles and expectations encouraged successful inter-disciplinary/inter-agency work. One of the psychologists noted that the situational analysis framework was very useful as it allowed her to clearly explain her interpretation of what was happening in the situation. Another psychologist said that there was 'a risk that different perspectives on a situation could cause conflicting opinions' but that 'frequent communication' and 'collaboration' allowed her to work better with other professionals and generate 'a greater chance that different specialists can negotiate the best form of assessment and planning appropriate to the situation for the child, leading to better outcomes'.

The participants reported eight supporting aspects of the frameworks which they used in inter-disciplinary/interagency work (Graph F). Three of the six psychologists reported finding collaboration supportive, while two of the six reported finding consultation supportive; the six other supportive aspects were reported once. 


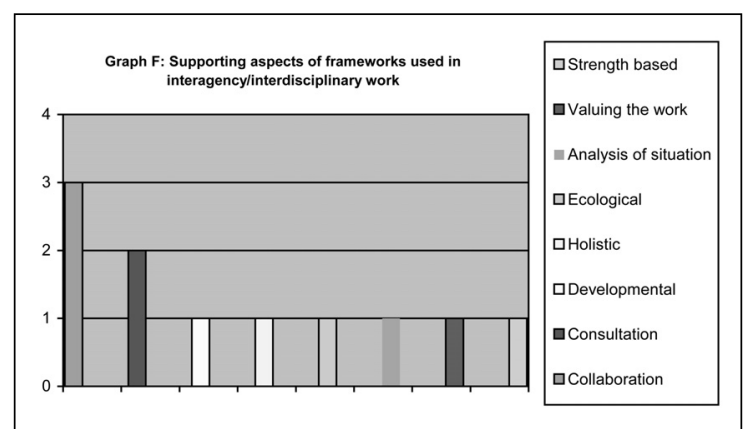

\section{DISCUSSION}

\section{Common reasons for referral}

The most commonly reported reasons for referral for severe and challenging behaviour were violent verbal or physical behaviour, non-compliance and anxiety, with social or learning difficulties reported only once. It appears from this data that schools are referring and GSE are prioritising those referrals which are focused on violent behaviour rather than those that focus on loss of social opportunities and learning. In its definition of severe and challenging behaviour the Ministry includes behaviour which affects 'social interactions and learning' (Ministry of Education, Severe Behaviour, 2008, p.18). The reasons for this are doubtless varied and could include the focus of the supporting literature of EIBC training on behaviour and behavioural theorists, the backgrounds, ages and length of experience of the individual participants, the kinds of referrals which schools make to MOE:SE, who is able to make referrals to MOE:SE, and the need for MOE:SE to prioritise referrals.

\section{Frameworks for practice}

The three most commonly mentioned frameworks for practice involving children and young people who have severe and challenging behaviour were EIBC, Situational Analysis and FBA. Some of the participants of the study specifically noted that they used FBA as part of their data-collection phase and that it helped them to define dimensions or aspects of a situation. It is interesting that they used FBA as a tool to help them as they worked within the situational analysis framework. The popularity of EIBC and FBA may be due to the recent training in EIBC which psychologists working at MOE:SE have undergone in the past two years.

Service pathways are an overarching framework specific to MOE:SE which is an organisational expectation for service standards as is RIBS, a review tool for ensuring service standards are adhered to in groups of fieldwork rather than individual fieldwork. The selection of a wide variety of frameworks for practice by the psychologists in the $50+$ age group may be correlated to their average length of service (11 years) and consequent exposure to a wide variety of frameworks. It may also be correlated to their varied cultural, work and educational backgrounds.

\section{Theoretical underpinnings}

The unanimous choice of behavioural theories to underpin the frameworks used is perhaps influenced by the recent training in EIBC. Behavioural theories are widely discussed in both the meta-analyses (Church, 2003, Meyer \& Evans, 2006;) which have informed the EIBC training. Behavioural theories are closely linked to FBA with Gresham, Watson and Skinner (2001) noting that operant learning theory underpins FBA.

Ecological theory, selected by five of the six participants, is noted by Annan (2005) as being both part of the style and structure of situational analysis. Other theories which are tied closely to the situational analysis framework are narrative, social learning and solution-focused theories. While Annan's article about situational analysis discusses some specific theories which informed the construction of the framework, the framework is flexible enough to allow the participants to bring their unique knowledge to the situation in order to achieve outcomes which are viewed by the participants as constructive.

\section{Supportive aspects of frameworks in inter- disciplinary/inter-agency work}

The most popular choice by practitioners for supportive aspects of their frameworks of practice in interdisciplinary/inter-agency work was collaboration. Annan (2005) directly addresses working with professionals from other disciplines and puts forward situational analysis as a way to enlist participants with multiple viewpoints as collaborators in the process of working towards new and positive outcomes. FBA provides a method of gathering data and presenting it, which facilitates understanding of why behaviour is occurring and highlights those areas which may be most likely to create beneficial change when intervened with.

The wide range of supportive aspects of frameworks for practice which were reported by participants may be reflective of their range of age, experience and backgrounds. Situational Analysis, EIBC and FBA frameworks appear to have been influential of the aspects which were reported, with positive reinforcement, collaboration and defining dimensions all being cited by more than one participant.

One of the most experienced psychologists reported that she was 'still learning' in response to the question about areas of frameworks for practice which needed development. This is perhaps reflective of both the recent training in EIBC which is currently being integrated into fieldwork and the nature of working with people who are individually unique and are involved in unique situations. It would be expected that aspects of the frameworks for practice be continually developing for the individual practitioner. Annan (2005) notes that both systems and individuals are diverse and that the situational analysis 
framework, which was reportedly used by four of the six psychologists, has been designed specifically to support fieldwork which is peculiar to each situation.

\section{Conclusion}

Data has been organised in this report to reflect the frameworks for practice most commonly reported by the participants, who were six psychologists working at MOE:SE, how these frameworks were supportive of a wide range of fieldwork and also specifically how they supported fieldwork which involves inter-disciplinary/ inter-agency work. Each of the more commonly named frameworks - EIBC, FBA and Situational Analysis - has been described and examined in relation to each other and the responses given by participants.

The responses given regarding frameworks and supporting theories could perhaps be described as diverse or, as some of the participants said, 'eclectic'. This may be reflective of the acknowledgement by MOE:SE that registered psychologists have a variety of backgrounds and training, and therefore use a variety of frameworks (Cull, personal communication, August $21,2008)$. It may also be reflective of the recent training in EIBC, which has taken place at MOE:SE and the educational programme leading to registration which is offered through Massey University which uses situational analysis as its framework. The registered psychologists in the 50+ age range selected the largest number of frameworks and this may reflect their experience with the profession and exposure to multiple frameworks.

It was interesting that the most common combination of reported frameworks used was EIBC, FBA and Situational Analysis. Perhaps this is because situational analysis is a broad enough framework to include the use of other frameworks as they are pertinent to the work. As the questions were asked specifically about working with severe and challenging behaviour, it might be expected that EIBC and FBA were frequently mentioned due to their applicability to working with severe and challenging behaviour. The limited number of participants of the study will limit the potential for generalisation of the results.

The research conducted has provided specific information about how some practising psychologists work within MOE:SE. The study has also been informative about how situational analysis can blend with the other frameworks for practice which are provided during in-service training at MOE:SE.

\section{REFERENCES}

Annan, J. (2005). Situational analysis: A framework for evidence-based practice. School Psychology International,. 26(2), 131-146.

Church, R.J. (2003). The definition, diagnosis and treatment of children and youth with severe behaviour difficulties: $A$ review of research. Report to the Ministry of Education. University of Canterbury, Education Department.

Cull, B. (2008). Personal Communication.

Gresham, F.M., Watson, T.S., \& Skinner, C.H. (2001) Functional behavioural assessment: Principles, procedures, and future directions. School Psychology Review. 30(2), 156-172.

Meyer, L.H. \& Evans, I. M. (2006). Final Report: Literature Review on Intervention with Challenging Behaviour in Children and Youth with Developmental Disabilities. Victoria University.

Ministry of Education, (2006). Specialist Service Standards. Wellington: Ministry of Education

Ministry of Education. Severe Behaviour Service: Retrieved September 28, 2008, from http://www.minedu.govt.nz/ educationSectors/SpecialEducation/ServicesAndFunding/ SevereBehaviourService.aspx

Monsen, J., Graham, B., Frederickson, N., \& Cameron, R.J.S. (1998). Problem analysis and professional training in educational psychology: An accountable model of practice. Educational Psychology in Practice. 13(4), 234-249.

Psychology Workforce: Summary Results from the 2006 Health Workforce Annual Survey. Retrieved August 29, 2008, from http://wwww.nzhis.govt.nz

\section{AUTHOR PROFILE}

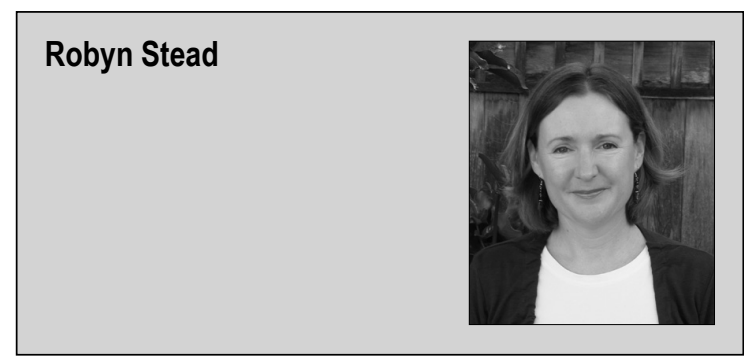

\section{Robyn Stead}

After teaching in the United States and New Zealand I completed my internship in Educational Psychology in 2008. Currently I am working with the Howick/Pakuranga Resource Teachers of Learning and Behaviour. I use situational analysis as my framework for practice and am enjoying the opportunities working as an RTLB to apply this framework. 